No 1 of a series on Public Health of STD control, edited by Graham Neilsen

\title{
Sexually transmitted diseases control in developing countries
}

\author{
Michael W Adler
}

Sexually transmitted diseases (STDs) are a major public health problem now compounded by the advent of AIDS and HIV infection. The size of the problem represented by STDs and HIV is unknown however it is estimated that there are 333 million new cases of STD per annum and currently 15-20 million people infected worldwide with HIV. Control programmes for STDs must prevent the acquisition of STDs, their complications and sequelae and interrupt and reduce transmission. They can also reduce the incidence of HIV infection. Such programmes must place emphasis on health education, condom usage, altering health seeking behaviour and providing case management. The syndromic approach currently offers the most realistic, and cost effective, way in which to treat patients.

(Genitourin Med 1996;72:83-88)

Keywords: STD; public health; control; developing countries

\section{Introduction}

The sexually transmitted diseases (STDs) represent a major public health problem within developing countries and the advent and increase of HIV infection during the last decade has highlighted the importance of infections spread by the sexual route. Failure to diagnose and treat traditional infections such as gonorrhoea, chlamydia and syphilis can have a deleterious effect on pregnancy and the newborn (such as miscarriage, prematurity, congenital and neonatal infections, blindness) and are common. Other complications, particularly in women, such as pelvic inflammatory disease, ectopic pregnancy, infertility and cervical cancer are large health and social problems.

Eighty per cent of HIV infection is spread by the sexual route ( $70 \%$ vaginal and $10 \%$ anal intercourse). The recognition that the presence of an STD, particularly genital ulcer disease (GUD) can enhance both the acquisition as a portal of entry and transmission of HIV by increased shedding of the virus within and from the genital tract has resulted increasingly in the development of integrated control programmes for HIV and STDs. Over and Piot $^{1}$ have reported that $70 \%$ of $\mathrm{HIV}$ infection in Africa is found in patients with an STD with lower, but increasing, levels of $15-30 \%$ in Thailand amongst STD patients.

Both AIDS and STDs have a major demographic, economic, social and political impact particularly in sub-Saharan Africa and increasingly in Asia. The World Bank ${ }^{2}$ in 1993 estimated that for those aged 15-44 years, STDs (excluding HIV) were the second cause of healthy life lost in women after maternal morbidity and mortality. In men HIV ranks first and considerably higher than STDs. Solutions to these diseases do not lie exclusively or currently to any major extent in the development of cures and vaccines. Control requires a broad based approach which addresses prevention, low cost/technology approaches to diagnosis, treatment and care, often delivered by non-medical personnel in rural settings.
The social, demographic and economic consequences of AIDS and STDs need addressing since they have major consequences on productivity, social and family structures. It follows from this that the medical model is too limited in controlling AIDS and STDs. It is pointless to develop strategies for the control of STDs and AIDS if appropriate systems and public sector financing are not in place for the delivery and adoption of services, health education, new technologies etc. The institutional and infrastructural issues of public sector financing, community development and capacity building are pivotal to any control programme.

This article, whilst recognising the essential nature of an integrated STD and HIV control programme and importance of infrastructural issues, will limit itself to models of care around STDs.

\section{Size of the problem}

(a) Worldwide Most developing countries do not have an effective control programme and notification system for STDs and HIV infection. The World Health Organisation (WHO) has been responsible for a series of estimates of the size of the problem represented by STDs and HIV infection. It estimates an annual total of 333 million new STD infections per annum. This does not include genital papilloma virus infection which WHO has in the past estimated at 30 million new infections per year, herpes at 20 million, and chancroid at 7 million (table 1). The major focus for STDs is south east Asia with an estimated 150 million new cases in 1995, and sub-Saharan Africa with 65 million. WHO projections for HIV infection show a current range of 15-20 million cumulative infections worldwide rising to $30-40$ million by the year 2000 (table 2). The Global AIDS Policy Coalition ${ }^{3}$ has also attempted to estimate the future size of the problem and calculates an upper range of 109 million people infected by 2000 . 
Table 1 The annual total of sexually transmitted infections (excluding HIV)

\begin{tabular}{ll}
\hline Condition & Total (million) \\
\hline Trichomoniasis & 170 \\
Genital chlamydia & 89 \\
Gonorrhoea & 62 \\
Syphilis & 12 \\
Total & 333 \\
\hline
\end{tabular}

Also genital papilloma virus 30 million, genital herpes 20 million, chancroid 7 million.

Source: WHO, 1995

Table 2 Adult HIV projections 1995-2000 (millions)

\begin{tabular}{lcc}
\hline & 1995 & 2000 \\
\hline WHO & $15-20$ & $30-40$ \\
AIW & $8-17$ & $38-109$ \\
(a) Sub-Saharan Africa & $5-11$ & $21-34$ \\
(b) South East Asia & 1 & $11-45$ \\
(c) North America & $1-1 \cdot 5$ & $1 \cdot 8-8$ \\
\hline
\end{tabular}

From AIDS in the World 1992 Global AIDS Policy Coalition.

(b) Nationally The lack of good data and notification systems has often been overcome by prevalence studies in particular countries. Such information is useful but limited since it is not totally representative of the population as it is obtained from atypical, high risk and usually consulting groups of individuals and/or patients. Table 3 indicates the prevalence of the common STDs in certain selected countries and settings. In commercial sex workers (CSWs) the prevalence of gonorrhoea can reach $50 \%$, and the prevalence of syphilis ranges from $23-32 \%$ for acute or previous infection. Levels of Chlamydia trachomatis can be as high as $25 \%$. In the developing world, prostitution is a driving force for STDs and HIV; for example in Kenyan urban STD clinics, $60 \%$ of men with a gonococcal urethritis or chancroid reported commercial sex exposure as the probable source of infection. Other high risk groups studied are men and women attending STD clinics, and, as expected, levels of infection are found to be high. Unfortunately, levels of infection can also be high in, what can be termed as "low-risk" groups, namely women attending ante-natal clinics. One finds very high levels of gonococcal and chlamydial infection from various studies carried out in Africa, Latin America and Asia. Rates are highest in sub-Saharan Africa, and lower in Latin America and Asia.

The STDs are important because of their complications and social stigma. The most important occur in women and are pelvic

Table 3 Prevalence of selected sexually transmitted diseases in developing countries

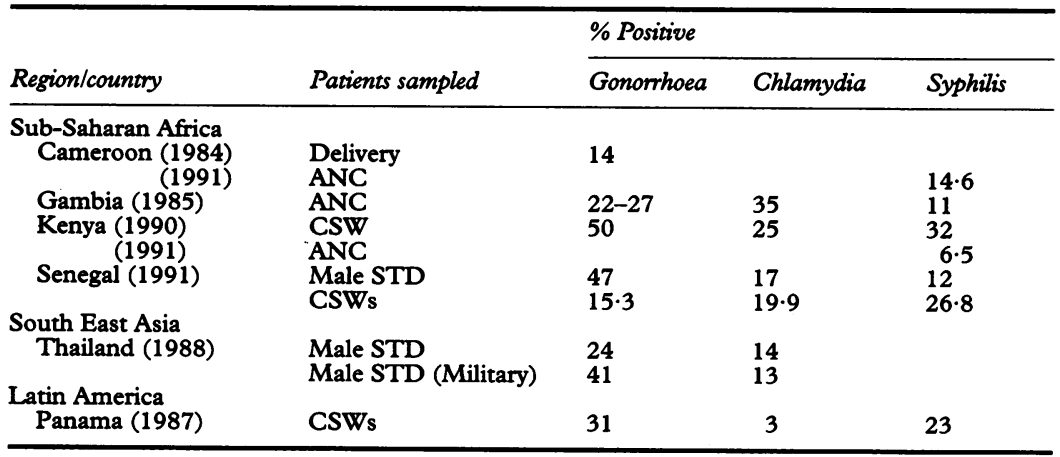

Table 4 Prevalence of selected STDs and their effect on pregnancy associated conditions (Nairobi)

\begin{tabular}{lccc}
\hline & $\begin{array}{c}\text { Neisseria } \\
\text { Gonorrhoea }\end{array}$ & $\begin{array}{c}\text { Chlamydia } \\
\text { trachomatis }\end{array}$ & $\begin{array}{c}\text { Treponema } \\
\text { pallidum }\end{array}$ \\
\hline $\begin{array}{l}\text { Prevalence } \\
\begin{array}{l}\text { Stillbirth } \\
\text { Prematurity }\end{array}\end{array}$ & $6 \%$ & $7 \%$ & $3 \%$ \\
$\begin{array}{l}\text { Postpartum pelvic } \\
\text { inflammatory disease }\end{array}$ & 2.9 & - & $5 \cdot 3$ \\
$\begin{array}{l}\text { Ophthalmia } \\
\text { Neonatorum }\end{array}$ & $42 \%$ & $31 \%$ & $\mathrm{n} / \mathrm{a}$ \\
\hline
\end{tabular}

Sources: Plummer, Laga, Elliot, Temmerman.

inflammatory disease (salpingitis), and ectopic pregnancy, but the infections also increase the risk of stillbirth and prematurity and affect the neonate (table 4). In sub-Saharan Africa 50\% of cases of infertility can be attributed to prior tubal infection, usually with gonorrhoea or chlamydia. Infertility is a personal and family tragedy that can lead to social isolation and ostracism of women, and eventual divorce.

Most developing countries have by now undertaken HIV seroprevalence studies, particularly among CSWs, intravenous drug users, and pregnant women. High levels of infection in these groups are found in most sub-Saharan Africa and south-east Asian countries.

\section{Control programmes}

Models for control programmes will vary between developed and developing countries and within countries. However, two basic principles are common to all programmes: (a) prevent the development of STDs, their complications and sequelae, (b) to interrupt and reduce transmission. These aims can be achieved or attempted through the two approaches of primary and secondary prevention (table 5).

1. Primary prevention Clearly it is best to avoid infection in the first place. The advent of AIDS has been a major influence in the increased awareness of the need for health education and the public acceptance of explicit messages and images. Programmes aimed at safer sexual practices and increased condom use have been widely advocated and implemented in both developed and developing countries. The success of such programmes is often difficult to assess and sadly initial modifications in behaviour are not always maintained over longer periods of time. This has been seen within both the United Kingdom (UK) and United States where initial and profound changes in sexual behaviour in homosexual men led to lower levels of STDs but unfortunately this alteration in behaviour has not been universally sustained.

Table 5 Strategy for control of STDs

Primary prevention
Health education/IEC to alter sexual behaviour and risk
Provision of condoms at affordable prices
Secondary prevention
Promotion of health care seeking behaviour
- Ease management of people with STD
through case finding and screening


Programmes to market and encourage the use of condoms have been at the heart of many control programmes and are particularly useful since they reduce acquisition and transmission of both traditional STDs and HIV infection. In Africa there has been encouraging evidence that increased condom use has been accepted by high risk groups such as CSWs and their clients and that this has altered levels of infection. Examples of this have been reported in Africa and SE Asia. A three year programme of condom promotion and STD control programme in Zaire saw an increase in consistent condom use from $0 \%$ to $68 \%$ amongst CSWs. ${ }^{4}$ Social marketing of condoms in Zaire saw sales increasing from 20000 to 18 million in a period of three years. ${ }^{5}$ Multiple outlets were used such as street traders, night clubs, CSWs and pharmacists. In parallel with the increased condom use in CSWs the incidence of gonorrhoea, trichomoniasis, genital ulcer disease and HIV declined. In Zimbabwe a community intervention at a cost of $\$ 85,000$ resulted in the distribution of $5 \cdot 7$ million condoms with a suggested resulting decline in STDs ranging from 6 to $50 \%$ in different areas. CSWs were found to have increased condom usage from $18 \%$ prior to the programme to $72 \%$ with their last client. $^{2}$ In Thailand an education programme advocating the use of condoms by CSWs and clients was followed by increased usage from a baseline of $14 \%$ to $94 \% .^{6} \mathrm{~A}$ concurrent decline in bacterial STDs was seen over the same time period.

Even though these results are promising it should not be forgotten that experience in developed countries suggests such changes are hard to sustain. Repeated reinforcement and monitoring is required. Also, as previously mentioned, social and economic issues need to be addressed. Often women are so poor and disempowered that they have sex on a commercial basis against their will and are unable to effectively negotiate the use of condoms by clients. Economic programmes may be required that help reduce the necessity for women to work as or supplement incomes by prostitution. At the same time women need to be taught skills that help them negotiate safer sex with clients and regular partners. This is particularly difficult with the latter since husbands and regular partners can see such negotiations as a reflection on themselves at the same time as suggesting that the woman has herself had multiple partners. The recognition of the substantial shift in cultural attitudes that are still necessary to establish wider condom use has led research workers to explore the use of vaginal virucides. Such an agent (or agents), if effective, would allow women more control. Raising the status of women in the developing world would be a crucial factor in the control of STDs and HIV, and can only be achieved through equality in the fields of education and employment.

Evaluation of programmes is required but all too often this is not carried out in a robust scientific manner. Oakley and colleagues ${ }^{7-8}$ carried out an extensive literature review of such programmes identifying 726 studies throughout the world. Eighty seven of the studies evaluated with a specific HIV/AIDS focus of which only seven were judged by the reviewers to be methodologically adequate using the criteria of the use of a control group, providing pre- and post-intervention data and reporting on all relevant outcomes.

The existence of Health Education and Information, Education and Communication (IEC) programmes does not necessarily mean that they are effective. Controlled trials of IEC are now being mounted, such a trial is currently being undertaken in Uganda. Inevitably there is a tension between the need to set up programmes "now" in an attempt to stem the tide of rising infection and that of trials proving effectiveness. A robust scientific approach is understandable but at the same time it must be remembered that new STDs and HIV infection are increasing at an alarming rate and that many successful interventions (for example smoking in developed countries) have never been properly evaluated but despite this are adjudged to be successful.

Health education and IEC need to be available to all sexually active adults but also importantly to young people prior to their first act of sexual intercourse. As well as general IEC special programmes particularly aimed at high risk or core groups are required. The messages may be different in content, emphasis
Figure 1 Health care seeking behaviour -women with STDs.

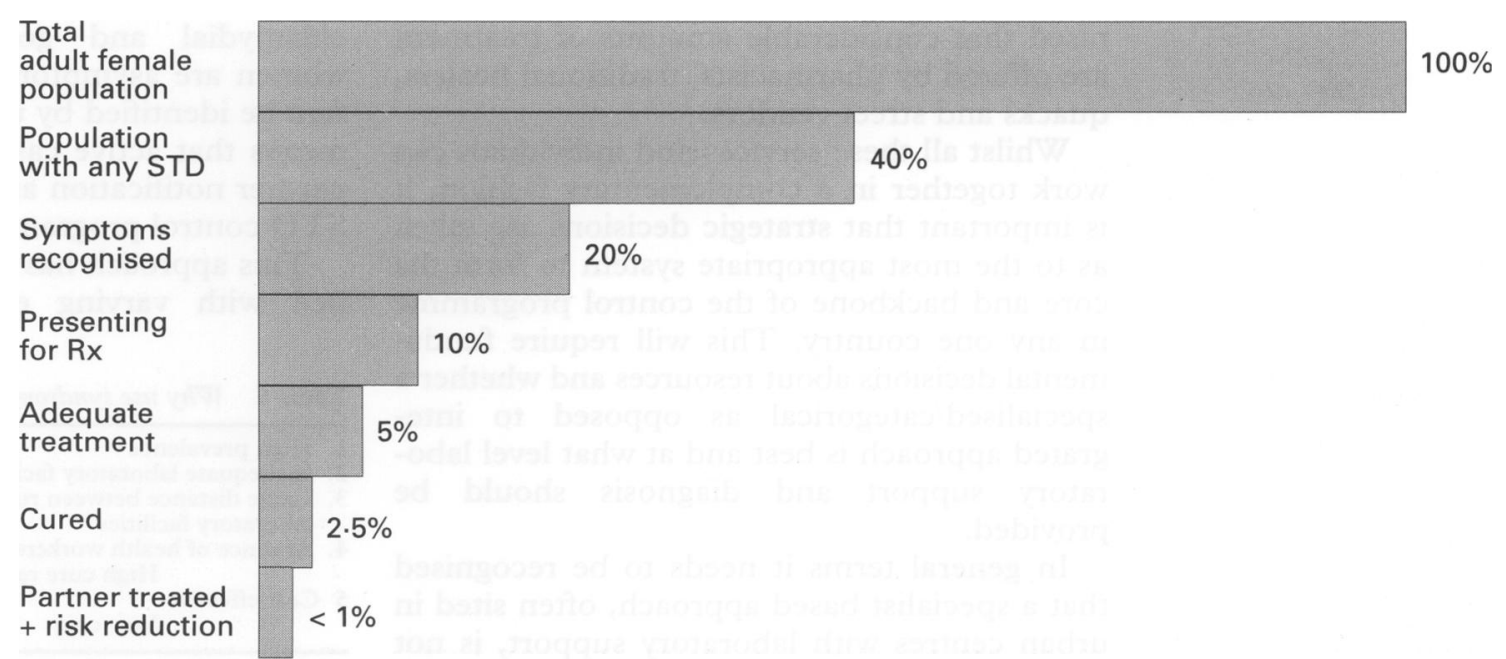


and delivery. For example many projects are being set up to deal with core groups such as CSWs, their clients and truck drivers. This work is often carried out in brothels, on the streets and at lorry stops.

\section{Secondary prevention}

(a) Healthcare seeking behaviour It is self evident, but often overlooked, that providing screening and diagnostic services however good and client friendly are of no use within a control programme unless utilised. It is an essential part of a health education programme to point out the symptoms associated with a possible STD but also to reinforce an understanding that many diseases are asymptomatic, particularly in women. It should be pointed out that ideally once symptoms develop further sexual contact should cease and care be sought and that those who are at high risk (for example multiple partners) should, regardless of symptoms, seek regular screening. Piot and Fransen have highlighted this basic issue of health seeking behaviour (fig 1). Their useful cascade illustrates that only half of the women with an STD symptom recognise its significance and only half of these present for treatment, of whom a further half receive adequate treatment. Health seeking behaviour is influenced by a large number of factors apart from knowledge and awareness. Economic structural adjustment programmes have been criticised since the introduction of fees for medical care, which are forced upon governments to fulfil these programmes, affects uptake of services. ${ }^{9}$ A documented example of this occurred in Nairobi's large STD clinic where the introduction of fees was followed by a reduction in attendance of $60 \%$ among men and $35 \%$ for women. ${ }^{10}$

(b) Case management Concepts and models of case management used in resource rich countries are usually inappropriate for implementation in developing countries in which such care is provided through a whole array of services and individuals. This will include specialised STD clinics, general hospital outpatients, primary healthcare centres (PHCs), maternal and child health facilities, family planning clinics, private practice. Few of those providing this care have specialist training or are medically qualified and it has to be recognised that considerable amounts of treatment are offered by pharmacists, traditional healers, quacks and street vendors.

Whilst all these services and individuals can work together in a complementary fashion, it is important that strategic decisions are taken as to the most appropriate system to form the core and backbone of the control programme in any one country. This will require fundamental decisions about resources and whether a specialised/categorical as opposed to integrated approach is best and at what level laboratory support and diagnosis should be provided.

In general terms it needs to be recognised that a specialist based approach, often sited in urban centres with laboratory support, is not available or appropriate and cost effective. Such specialist centres are expensive and only reach small segments of the population. The WHO has placed increasing emphasis on integrated approaches, especially at PHC level, using a syndromic approach for patient management. ${ }^{11}$ This approach recognises the limitation of resources for health care and specialist trained medical personnel (table 6). It is advocated for use particularly in high prevalence areas, where there are inadequate laboratory facilities and trained staff. A very recent, important and exciting study from Tanzania has demonstrated the importance of an integrated STD programme in rural communities on HIV incidence. ${ }^{12}$ This randomised controlled trial showed that improved STD care integrated at primary care level resulted in a reduction of HIV incidence of $42 \%$ over the two year period of the study. The study confirms the effect of STDs on HIV acquisition and transmission and that the integrated nonspecialist and syndromic approach can be very effective.

The syndromic approach uses algorithms based upon commonly presenting signs and symptoms, for example genital ulcer, urethral and vaginal discharge, where laboratory support may or may not be present (fig 2). The algorithm for genital ulcers works on the basic premise that the two commonest causes of such a sign/symptom are syphilis and chancroid and that with no laboratory support and the difficulty in establishing an accurate clinical diagnosis (see later) it is appropriate to give therapy for both conditions. Counselling, education, condom use and partner notification are encouraged. Likewise the algorithm for urethral discharge assumes that the aetiology could be gonococcal and/or chlamydial and that therapy appropriate for both should be utilised. Both these algorithms for ulcers and urethral discharge have been evaluated and been shown to be robust and sensitive (see below). Unfortunately the algorithm for vaginal discharge is not so discriminating since this symptom is so common, non-specific with multiple possible non-sexually-acquired aetiologies. Attempts to improve this algorithm have been made by incorporating a risk assessment which includes symptoms in the partner, number of partners, age and marital status.

It is also recognised that the majority of chlamydial and gonococcal infections in women are asymptomatic and will not therefore be identified by use of an algorithm. This means that active case finding, screening and partner notification are vitally important in an STD control programme aimed at women.

This approach has been adopted and modified with varying enthusiasm by different

Table 6 Why use syndrome based approach?

1. High prevalence

2. Inadequate laboratory facilities in rural areas

3. Large distance between rural PHC centres and specialised laboratory facilities

4. Absence of health workers with training

High cure rate

5 Cost effective

Low cost 
Figure 2 Algorithms for various syndromes.

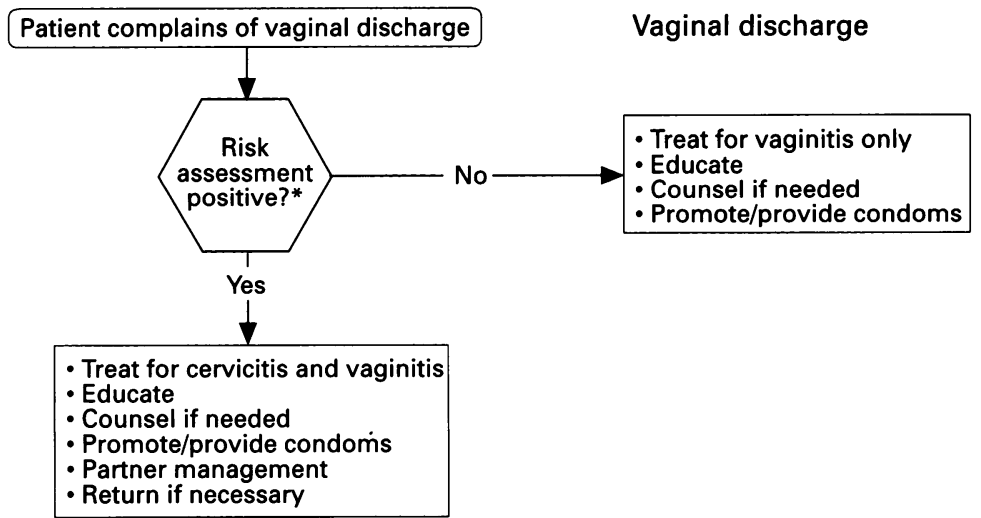

*Positive = partner symptomatic or any two of: age $<21$ years; single; $>1$ partner; new partner in past 3 months
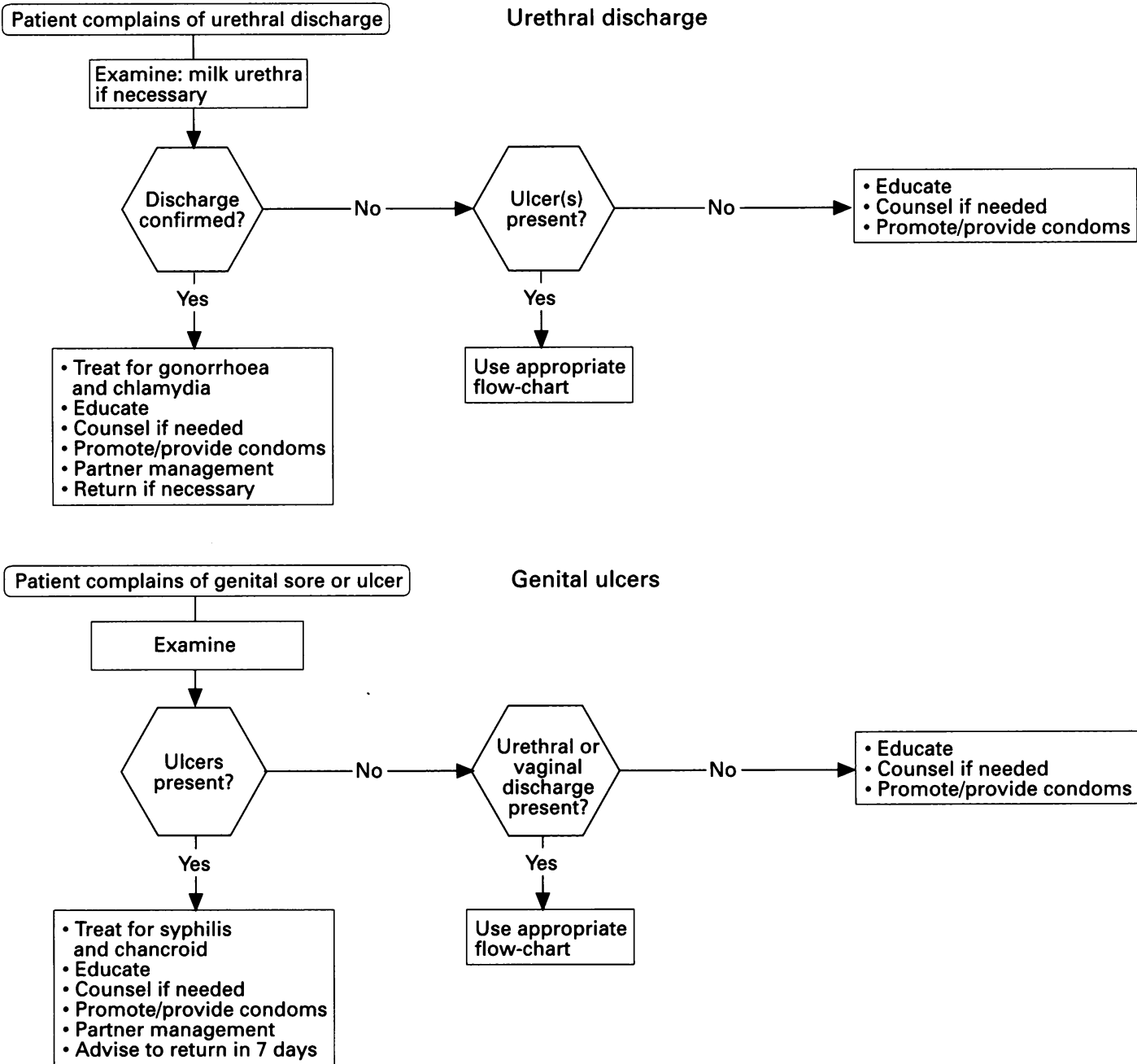

countries who will add in their own treatment schedules. Clinicians brought up with the philosophy that treatment only follows a full microbiological diagnosis have often found this approach hard to accept and consider it to be unscientific. ${ }^{13}$ In the last few years a number of studies have attempted to evaluate the effectiveness and cost of the syndromic approach. Grosskurth, Mayaud and colleagues (private communication) have reported their work evaluating the cure rate using the syndromic approach in 25 health units in Tanzania over a period of 550 observation months. They looked at cure rates using standardised treatments for patients presenting with urethral/vaginal discharge, genital ulcer and lower abdominal pain. The total reported cure rate using this approach was $81 \%$.

A study carried out in Kigali, Rwanda compared the sensitivity of the three approaches to the diagnosis and management of genital ulcers which could be due either to syphilis and/or chancroid. ${ }^{14}$ All 395 patients entered into the study had cultures performed for herpes, Haemophilus ducreyi and serological tests for syphilis (RPR and TPHA). The algorithms were (1) in settings without laboratory support, (2) a hierarchical one where an RPR or dark field microscopy could be performed and finally (3) clinical diagnosis based on the doctor's clinical diagnosis without laboratory back-up. In practical terms this meant that 
patients managed with algorithm (1) would be given treatment for both syphilis and chancroid, with algorithm (2) treatment was given as appropriate depending on the laboratory confirmed diagnosis and for (3) (clinical diagnosis) treatment as indicated on the clinical diagnosis alone without laboratory tests being available.

The sensitivity of these models varied. The range was from $98 \%$ (Algorithm 1) to $18 \%$ (clinical diagnosis). From a public health perspective a high sensitivity of a diagnostic approach is more important than a high specificity. However, the use of the most sensitive approach (algorithm 1) does mean that people are being treated for both chancroid and syphilis when they may only be suffering from one condition.

A major problem of the syndromic approach, as mentioned previously, is that patients with asymptomatic infection are not detected and treated; this is of particular importance in women. Additionally, in women the algorithm for vaginal discharge has been found to be poorly predictive of an STD. Vuylsteke and Laga ${ }^{15}$ have attempted to evaluate the use of algorithms among women (pregnant women and prostitutes) who present for other than STDs, for example are attending antenatal and family planning clinics. Their first algorithm required no examination and symptoms were the only basis for treatment and second algorithm additionally included examination. The third algorithm included a speculum examination, swab test of cervical discharge (positive if yellow mucopurulent endocervical discharge), a quantified leucocyte esterase urine dipstick and scoring system of risk. The results indicated that the relationship between classical symptoms and signs with the eventual diagnosis of a gonococcal and/or chlamydial infection was weak when evaluated in both high (prostitutes) and low (pregnant women) prevalence populations. The use of a scoring system which includes risk markers as well as symptoms and signs gave a better sensitivity but a specificity that was essentially comparable to the first two algorithms. However, it did not require a time consuming and often logistically impossible speculum examination. Considerable refinement of vaginal discharge algorithms is still required.

At the same time the challenge exists to find a rapid, reliable and inexpensive diagnostic test that can be used for widescale screening purposes without resorting to invasive tests such as speculum examination and urethral swabs. Considerable effort is now being made to develop such new technologies especially using urine.

\section{Conclusions}

STDs are increasingly important diseases with considerable morbidity. Their control in developing countries has to be given priority while at the same time recognising that programmes must be cheap and pragmatic. The development of good widely disseminated IEC and training staff in the use of the syndromic approach are the two most important planks of a good programme. Such a programme can be integrated with HIV/AIDS and/or maternal and child health programmes depending on local circumstances and attitudes. It is essential that the size of the problem facing us does not lead to total inertia and total mesmerisation.

1 Over M, Piot P. HIV Infection and Sexually Transmitted Diseases. HSPR 26, Washington, The World Bank, 1991. 2 The World Bank. World Development Report 1993 Investing in Health. New York, Oxford University Press, 1993

3 Mann J, Tarantole DIM, Netter W, eds. AIDS in the World. Harvard, Harvard University Press, 1992.

4 Laga M, Alary M, Nzila N, et al. Condom promotion, sexually transmitted diseases, treatment and declining incidence of HIV infection in female Zairian sex workers. Lancet 1994;344:246-8.

5 Kyungu Mormat E. Condom and social marketing and mass media in Zaire. In: proceedings of meeting on effective approaches to AIDS prevention. Geneva, WHO, 1992 .

6 Hanenberg RS, Rojanapithayakorn W, Kunasol P, Sokal DC. Impact of Thailand's HIV control programme as indicated by the decline of sexually transmitted diseases. Lancet 1994;344:243-45.

7 Oakley A, Fullerton D, Ackland J, et al, 1994. Towards effective intervention: a critical evaluation of HIV prevention and sexual health education interventions. MRC and HEA report.

8 Oakley A, Fullerton D, Holland S, et al. Sexual health education interventions for young people: a methodological review. BMҰ 1995;310:158-62

9 Lurie P, Hintzen P, Lowe RL. Socioeconomic obstacles to HIV prevention and treatment in developing countries: the roles of the International Monetary Fund and the the roles of the International Mon

10 Moses S, Manji F, Bradley JE, et al. Impact of user fees on attendance at a referral centre for sexually transmitted diseases in Kenya. Lancet 1992;340:463-6.

11 World Health Organisation. Management of patients with sexually transmitted diseases. Report of a WHO Steering Group 1991. WHO technical report series 810 . Geneva.

12 Grosskurth H, Mosha F, Todd J, et al. Impact of improved treatment of sexually transmitted diseases on HIV infection in rural Tanzania: randomised control trial. Lancet 1995;346:530-6.

13 Kumar B, Handa S, Dawn G. Syndromic management of genital ulcer disease-a critical appraisal. Genitourin Med 1995;71:197.

14 Bogaerts J, Vuylsteke B, Alary M, et al. Simple algorithms for the management of genital ulcers: an evaluation in primary health care centre in Kigali, Rwanda. Clin Infect Dis (in Press)

15 Vuylsteke B, Laga M, Alary M, et al. Clinical algorithms for the screening of women for gonococcal and chlamydial infection. Evaluation of pregnant women and prostitutes in Zaire. Clin Infect Dis 1993;17:82-8. 
demonstrate typical features. Rare conditions in which only a handful of cases have been described are also covered.

The book is aimed at dermatologists, but many conditions are the cutaneous manifestations of systemic infections and should be of interest to a wider "audience". Atypical presentations of common skin complaints frequently occur in HIV infection, and these are splendidly illustrated together with HIV specific conditions. Many rare conditions look very similar and the message seems to be: if in doubt, biopsy-the differential diagnosis may be wider than you think.

SARAH EDWARDS
International Congress of Sexually Transmitted Diseases

12th Meeting of the International Society for Sexually Transmitted Diseases Research (ISSTDR) held jointly with the International Union Against Venereal Diseases and Treponematoses (IUVDT) 19-22 October 1997, Sevilla, Spain.

For further information contact: ICSTD
Congress Secretariat, Apdo 6077, 41080Sevilla, SPAIN. Fax. 95.4377413.

\section{CORRECTION:}

In the paper STD control in developing countries by $M$ W Adler (Genitourin Med 1996;72:83-8) it should have been stated that the source of figure 1 was P Piot and L Fransen.

\section{NOTICES}

The International Society for Cutaneous Lymphomas

1 st Consensus Conference 14 June, HiltonHotel, Sydney. Contact: R Dummer, M D, Department of Dermatology, Gloriastr. 31, $\mathrm{CH}-8091 \mathrm{Zurich} /$ Switzerland.

\section{DIARY OF EVENTS}

3-6 July 1996

7-12 July 1996

11-14 Sept 1996

15-18 Sept 1996

18-20 Sept 1996

3-7 Nov 1996

1-5 Dec 1996
Fifth International Drug Resistance Workshop. Whistler, Canada. International AIDS Conference, Vancouver, Canada.

Third European Chlamydia Meeting, Vienna, Austria.

Interscience Conference on Antimicrobial Agents and Chemotherapy (ICAAC). New Orleans, USA.

Society for Social Medicine. Dundee, UK.

3rd International Congress on Drug Therapy in HIV Infection. Birmingham, UK

International Papillomavirus Workshop, Queensland, Australia. 